\title{
Simplified input-output inversion control of a double pendulum overhead crane for residual oscillations reduction
}

\author{
Marco Giacomelli $^{\S} \quad$ Fabrizio Padula ${ }^{\mathbb{I}} \quad$ Luca Simoni $^{\S} \quad$ Antonio Visioli $^{\S o}$ \\ $\S$ Dipartimento di Ingegneria Meccanica e Industriale \\ University of Brescia, Italy \\ e-mail: \{m.giacomelli009,1.simoni002,antonio.visioli\}@unibs.it \\ ${ }^{o}$ corresponding author

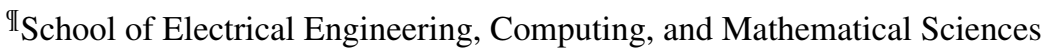 \\ Curtin University, Australia \\ e-mail: fabrizio.padula@curtin.edu.au
}

This is the pre-peer reviewed verion of the following article: Simplified inputoutput inversion control of a double pendulum overhead crane for residual oscillations reduction, which has been published in final form at 10.1016/j.mechatronics.2018.10.002.

This article may be used for non-commercial purposes in accordance with Journal terms and conditions for Self-Archiving.

\begin{abstract}
In this paper we present the application of an input-output inversion technique for the open-loop control of an overhead crane modelled as a double pendulum. The method is mathematically derived, obtaining a parametric trajectory that ensures reduced residual oscillations. Then, it is shown that the postactuation can be neglected so that the method can be implemented with standard industrial drives. The robustness of the method is evaluated by means of simulations, and the performance of the method is experimentally compared with the well-known input shaping technique. The advantages of using a double pendulum model instead of a simple pendulum one are also shown.
\end{abstract}

Keywords: Overhead crane, control, residual oscillation, trajectory planning, inputoutput-inversion. 


\section{Introduction}

Overhead cranes are widely used in industry for their capability of moving heavy payloads despite their relative simplicity and low cost. Much research effort has been spent in the last thirty years [1] in devising suitable controllers for these systems, in order to increase their safety and productivity. In fact, the main control requirement, in addition to minimizing the travelling time or the energy [2-4], is to reduce the residual oscillation which is in general a difficult task for a human operator.

Many of the different proposed methodologies are related to the closed-loop control of the system, in which the angle of the payload is measured. In this context, for example, a Lyapunov approach [5, 6], a fuzzy logic mechanism [7]-9], the model predictive control concept [10-12], a gain scheduling technique [13, 14], sliding mode approach [15, 16] and adaptive schemes [17] have been exploited.

However, it has to be recognized that the simplest approach is to apply an openloop controller, that is, a motion planning method for which the cart motion is determined in order to obtain an efficient motion of the payload [18]. This solution is the most appreciated in the industrial context because it avoids the use of a sensor to measure the angle of the rope (which is unlikely to be available in practice), it can be easily implemented with standard off-the-shelf motion control devices (where complex optimization methods cannot be implemented) and it is fully compatible with the typical (velocity) commands usually performed by crane operators. In this framework, the most well-known methodology, which has proved to be very effective in practical cases, is the input shaping [19--28]. This consists in convolving the motion of the cart, initially defined as if there are no oscillatory behaviours in the system, with a suitable sequence of impulses in order to generate the actual cart motion in such a way that no residual payload oscillation appears. Different modifications of the input shaping technique have been proposed in the last 10 years to improve robustness and to address practical application issues. Indeed, the input shaping can be implemented with standard hardware in such a way that it can help the operator to compensate for the residual vibration in a transparent way. This is often not straightforward with other motion planning methodologies such as, for example, [29] where an optimization strategy has been proposed.

It has to be recognized that, in many cases, considering the crane as a simple pendulum is not sufficient in order to obtain an accurate modelling, which is particularly relevant when open-loop techniques are implemented. Indeed, a double pendulum model can capture much more precisely the dynamics of an overhead crane in those cases where the mass of the hook and the distance between the centers of mass of hook and payload are not negligible. Another situation where the simple pendulum is not sufficient, is the case of distributed mass payloads, for which the lumped mass simplification hides the presence of a second oscillatory mode of the system.

For overhead cranes which exhibits double pendulum dynamics, closed-loop techniques have been proposed in the literature [30-34]. In particular, in [31] a robust 
nonlinear closed-loop control is proposed; in [32] a fuzzy approach is applied to the problem of oscillating payloads with double pendulum dynamics, while in [33] the sliding mode control is used. A super-twisting-based antiswing controller is proposed in [34]. However, these closed-loop solutions do not seem practical as, again, they require the measure of the load angle, which is not available in practice. Open-loop techniques have also been proposed for the antiswing control of cranes with double pendulum swing effects. The extension to multimode systems for the input shaping technique has been proposed in [35-38]. In [39], an optimal energy trajectory planner is proposed, while in [40,41] a minimum-time trajectory planning algorithm is presented. Both the optimization-based approaches include various states constraints and use the differential flatness of the crane dynamics to translate the constrained optimization problem into a convex optimization problem. However, the aforementioned open loop techniques, with the exception of input shaping, require the solution of non-trivial optimization problems. Solvers for optimization problems are not present in standard industrial components, and even if solvers can be implemented, the time required for the solution does not allow for the use of these techniques for online trajectory planning.

In this paper we propose an input-output inversion technique for the residual oscillation reduction, by considering both velocity and position control tasks. In particular, we devise our technique using a double-pendulum model that can capture the dynamics of distributed payloads, and we systematically validate the proposed approach through simulations and experimental results. Finally, we provide a fair comparison with the input shaping technique, thus allowing the user to ponder the pros and cons of each technique and to choose the most appropriate one. Note that the use of input-output inversion techniques has already been demonstrated to be effective for the control of oscillatory systems [42]. In particular, in [43] the input-output method has been employed in conjunction with an observer-based state-space controller while in [44] a simplified inversion methodology is proposed for the control of a single oscillatory mode overhead crane. However, both contributions rely on the single-pendulum model, which seems unrealistic and limits the practical applicability to non-distributed payloads. Moreover, the use of an advanced control paradigm and the lack of experimental results renders the technique proposed in [43] unsuitable for the practical implementation in industry, while in [44] the robustness issue is overlooked. Finally both contribution do not provide a comparison with other methodologies available in the literature.

The inversion-based design technique is based on a two steps procedure. First, a desired monotonic output function, with a required order of continuity and with a finite transition time $\tau$, is defined. For this purpose, a transition polynomial is an appropriate choice [45]. Secondly, the system dynamics is inverted to determine an input signal in such a way that, at least in the nominal case, the actual output is equal to the desired one. In this paper, the procedure is used to obtain the desired cart position or velocity once the oscillation-free desired motion of the payload has been defined. As such, the input-output inversion approach results in the synthesis of suitable reference (feedforward) signals. It is worth stressing that this approach 
do not require an ad hoc control architecture and for this reason it can be applied to standard off-the-shelf industrial controllers. This makes the methodology very suitable for a practical usage in industry. In fact, we use the standard feedback cascade PI control architecture to cope with the unavoidable model uncertainties and we determine in closed-form the analytical expression of the reference signals, making our approach suitable for the implementation on commercial devices. In addition, a feedforward torque signal for the cart motion can be determined in order to improve the performance of the cart control. In this regard, despite being deemed as an open-loop technique, the input-output inversion allows the full exploitation of the commercial closed loop-control control systems, thus combining the advantages of both approaches.

The main issue in the application of this design technique is that, because of the presence of a negative zero, the input signal and the state of the system do not attain the steady state value together with the system output at time $t=\tau$. In other words, a postaction results [46] and this implies that a control action has to be applied even when the payload has already attained its final position or velocity (depending on the motion control task).

Hence, in order to further improve the practical applicability of the devised methodology, we propose the use of a modified version of the input-output inversion technique where the postaction is neglected. After tailoring the general methodology to the overhead crane dynamics, we show that, when the pendulum damping ratio is negligible (as it is often the case in practical applications, and as normally assumed in the literature), the time constant of the exponential function that generates the postaction is also negligible. This greatly improves the industrial applicability of the proposed methodology. Indeed, the control action attains its steady-state value when the load reaches its final position/velocity, i.e., in a finite time $\tau$.

Eventually, we experimentally show the effectiveness of the proposed approach both for velocity and position control. The experimental laboratory scale setup is built using exclusively off-the-shelf components. In order to thoroughly assess the applicability of the methodology in an industrial environment, we also test its robustness against model uncertainties. In this context, we compare the proposed methodology to the input shaping technique, by analyzing pros and cons of both the approaches in terms of performance and robustness. In particular, we show that one of the main advantages of the inversion-based methodology is that, differently from the input shaping, the transient time of the payload velocity/position is not limited by the system natural oscillation period. This allows the full exploitation of the crane actuator. In fact, the transient time can be selected by the user by taking into account the actuator limitations and the trade-off between performance and robustness.

Compared with the existing techniques, the main contributions of the proposed approach can be summarized as follows:

1. all the trajectories and signals necessary to implement the proposed technique are polynomial, smooth and parametric, and they can be computed 
analytically in closed-form. The trajectory of the payload is monotonic by construction;

2. the proposed technique does not require optimizations to be solved, and can therefore be easily be implemented on standard industrial off-the-shelf components as demonstrated by the experimental results;

3. the proposed approach hinges on a single tuning parameter (the transition time $\tau$ ) that has a clear and immediate physical meaning, allowing the operator to easily handle the robustness/performance trade-off depending on the application.

This paper is structured as follows: in Section 2 the double pendulum model is obtained and in Section 3 the general idea of input-output inversion technique is briefly reviewed. In Section 4 the control scheme is presented, while in Section 5 the polynomial control laws are calculated by inverting the double pendulum model of the crane. Simulation results are presented in Section 6 , while in Section 7 the robustness of the method is compared with input-shaping techniques. In Section 8 , the experimental set-up is presented. Finally, experimental results are presented in Section 9 and concluding remarks are given in Section 10 .

\section{Double pendulum model}

As mentioned in the introduction, whenever the hook mass is not negligible and/or the payload is distributed, the single-pendulum model is not sufficient to accurately describe the dynamics of an overhead crane system. As such, we consider the twomass pendulum shown in Figure 1. The system consists of a concentrated payload and a hook with relevant mass. In the model, $x_{c}$ is the position of the cart, $u(t)$ is the force applied to the cart, $m_{C}, m_{1}, m_{2}$ are, respectively, the mass of the cart, of the hook and of the payload, $C_{C}, C_{1}, C_{2}$ are, respectively, the viscous friction

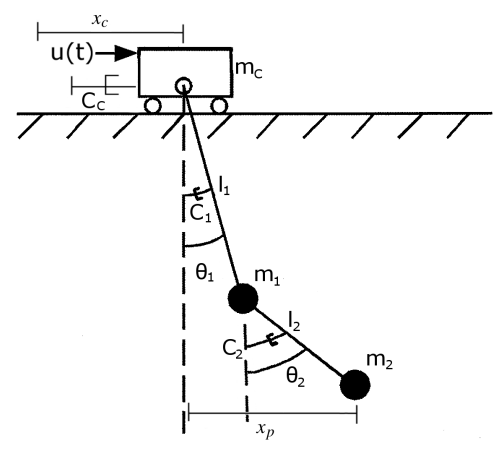

Figure 1: Sketch of an overhead crane, seen as a double pendulum connected to a sliding cart. 
coefficients of the cart, of the first and of the second cable, $\theta_{1}, \theta_{2}$ are the angles between the vertical and the first and second cable, while $l_{1}, l_{2}$ are, respectively, the lengths of first and of the second cable.

Note that the double pendulum model is very general. Indeed, in the case of distributed payload (with non-negligible moment of inertia), an equivalent dynamical two-mass system can be calculated. By considering the first mass as positioned on the upper extremity of the payload, the values of the masses $m_{1}$ and $m_{2}$ and the length $l_{2}$ are given by the solution of a system of three equations:

$$
\left\{\begin{array}{l}
m_{1}+m_{2}=M \\
m_{1} l_{g}=m_{2}\left(l_{2}-l_{g}\right) \\
m_{1} l_{g}^{2}+m_{2}\left(l_{2}-l_{g}\right)^{2}=I,
\end{array}\right.
$$

where $M$ is the total mass of the payload, $l_{g}$ is the distance of the center of gravity of the distributed payload to the top of the payload itself, and $I$ is its centroidal moment of inertia.

Note that the first equation ensures that the equivalent system has the same total mass of the distributed payload, the second one places the center of gravity of the equivalent two-masses system congruently to the position of the center of gravity of the distributed payload, and the third one ensures an equivalence of the centroidal moment of inertia.

On the other hand, a reduced simple pendulum model [1], starting from the model in Figure 1 1 , can be obtained by solving the system of two equations:

$$
\left\{\begin{array}{l}
M_{s}=m_{1}+m_{2} \\
L_{S} M_{s}=m_{1} l_{1}+m_{2}\left(l_{1}+l_{2}\right)
\end{array}\right.
$$

where $M_{s}$ is the mass of the payload and $L_{s}$ the length of the cable in the simple pendulum model. Note that the first equation ensures that both simple and double pendulum model have the same total mass, while the second equation ensures the same moment of inertia with respect to the center of rotation of the pendulum, that is the cart pivot.

In order to have a tractable model of the crane, we make the following standing assumptions:

- the mass of the cable is negligible compared to the mass of the payload;

- the movements of the system is on a $x-y$ plan, with no lateral swing, resulting in a 2D dynamic model.

- the rope is considered as an inflexible rod (this assumption is motivated by the fact that, in the normal movements of an industrial crane, the rope is always stretched and its elasticity is in fact negligible);

Remark. Note that the assumption of an infinite rigidity of the cable is very common in literature [1]. There are some special cases, see [47], where the effects of 
elasticity in the cables are non-negligible. However, these effects only appear with rare particularly heavy payloads, and impact on the lateral dynamics, which is out of the scope of this paper. In the vast majority of the applications, the standing assumption of rigid cable, which is also assumed in this paper, works perfectly.

The Lagrangian method can be used to find the differential equations that describe the dynamics of the system in Figure 1, obtaining:

$$
\left\{\begin{array}{l}
\left(m_{C}+m_{1}+m_{2}\right) \ddot{x}+\left(m_{1}+m_{2}\right) l_{1} \ddot{\theta}_{1} \cos \theta_{1}+m_{2} l_{2} \ddot{\theta}_{2} \cos \theta_{2} \\
-\left(m_{1}+m_{2}\right) l_{1} \dot{\theta}_{1}^{2} \sin \theta_{1}-m_{2} l_{2} \dot{\theta}_{2}^{2} \sin \theta_{2}=u(t)-C_{C} \dot{x} \\
\left(m_{1}+m_{2}\right) l_{1} \ddot{x} \cos \theta_{1}+\left(m_{1}+m_{2}\right) l_{1}^{2} \ddot{\theta}_{1}+m_{2} l_{1} l_{2} \ddot{\theta}_{2} \cos \left(\theta_{1}-\theta_{2}\right) \\
+m_{2} l_{1} l_{2} \dot{\theta}_{2}^{2} \sin \left(\theta_{1}-\theta_{2}\right)+\left(m_{1}+m_{2}\right) g l_{1} \sin \theta_{1}=-\frac{C_{1}}{l_{1}} \dot{\theta}_{1} \\
m_{2} l_{2} \ddot{x} \cos \theta_{2}+m_{2} l_{2}^{2} \ddot{\theta}_{2}+m_{2} l_{1} l_{2} \ddot{\theta}_{1} \cos \left(\theta_{1}-\theta_{2}\right) \\
-m_{2} l_{1} l_{2} \dot{\theta}_{1}^{2} \sin \left(\theta_{1}-\theta_{2}\right)+m_{2} g l_{2} \sin \theta_{2}=-\frac{C_{2}}{l_{2}} \dot{\theta}_{2} .
\end{array}\right.
$$

The model can be linearized around its sole stable equilibrium point, that is for $\theta_{1}=0$ and $\theta_{2}=0$ (and obviously zero velocities), obtaining the state-space representation

$$
\left\{\begin{aligned}
\dot{x}_{s s}(t) & =A x_{s s}(t)+B u(t) \\
x_{p}(t) & =C x_{s s}(t),
\end{aligned}\right.
$$

where

$$
\begin{aligned}
& x_{s s}=\left(\begin{array}{llll}
x_{c} & \dot{x}_{c} & x_{p} & \dot{x}_{p}
\end{array}\right)^{T}, \\
& A=\left(\begin{array}{cccccc}
0 & 1 & 0 & 0 & 0 & 0 \\
0 & -\frac{C_{C}}{m_{C}} & \frac{\left(m_{1}+m_{2}\right) g}{m_{C}} & \frac{C_{1}}{l_{1}^{2} m_{C}} & 0 & 0 \\
0 & 0 & 0 & 1 & 0 & 0 \\
0 & \frac{C_{C}}{l_{1} m_{C}} & -\frac{g\left(m_{1}+m_{2}\right)\left(m_{1}+m_{c}\right)}{l_{1} m_{1} m_{C}} & -\frac{C_{1}\left(m_{1}+m_{C}\right)}{l_{1}^{3} m_{1} m_{C}} & \frac{g m_{2}}{l_{1} m_{1}} & \frac{C_{2}}{l_{1} l_{2}^{2} m_{1}} \\
0 & 0 & 0 & 0 & 0 & 1 \\
0 & 0 & \frac{g\left(m_{1}+m_{2}\right)}{l_{2} m_{1}} & \frac{C_{1}}{l_{1}^{2} l_{2} m_{1}} & -\frac{g\left(m_{1}+m_{2}\right)}{l_{2} m_{1}} & -\frac{C_{2}\left(m_{1}+m_{2}\right)}{l_{2}^{3} m_{1} m_{2}}
\end{array}\right), \\
& B=\left(\begin{array}{llllll}
0 & \frac{1}{m_{C}} & 0 & -\frac{1}{l_{1} m_{C}} & 0 & 0
\end{array}\right)^{T}, \\
& C=\left(\begin{array}{llllll}
1 & 0 & l_{1} & 0 & l_{2} & 0
\end{array}\right) .
\end{aligned}
$$

From system (4) the transfer function of the system is determined as

$$
F(s):=\frac{X_{P}(s)}{U(s)}=\frac{N_{F}(s)}{D_{F}(s)}
$$

where

$$
N_{F}(s)=\left(C_{1} C_{2}\right) s^{2}+\left(C_{1} g m_{2} l_{2}^{2}+C_{2}\left(g l_{1}^{2} m_{1}+g l_{1}^{2} m_{2}\right)\right) s+g^{2} l_{2}^{2} l_{1}^{2} m_{2}\left(m_{1}+m_{2}\right)
$$


and

$$
D_{F}(s)=p_{6} s^{6}+p_{5} s^{5}+p_{4} s^{4}+p_{3} s^{3}+p_{2} s^{2}+p_{1} s,
$$

where

$$
\begin{aligned}
p_{6}= & l_{1}^{3} l_{2}^{3} m_{2} m_{1} m_{C}, \\
p_{5}= & C_{1} l_{2}^{3} m_{1} m_{2}+C_{2} l_{1}^{3} m_{1} m_{C}+C_{1} l_{2}^{3} m_{2} m_{C}+C_{2} l_{1}^{3} m_{2} m_{C}+C_{C} l_{1}^{3} l_{2}^{3} m_{1} m_{2}, \\
p_{4}= & g m_{C} l_{1}^{3} l_{2}^{2} m_{1} m_{2}+g m_{C} l_{1}^{3} l_{2}^{2} m_{2}^{2}+C_{2} C_{C} l_{1}^{3} m_{1}+C_{2} C_{C} l_{1}^{3} m_{2}+g l_{1}^{2} l_{2}^{3} m_{1}^{2} m_{2} \\
& +g l_{1}^{2} l_{2}^{3} m_{1} m_{2}^{2}+g m_{C} l_{1}^{2} l_{2}^{3} m_{1} m_{2}+g m_{C} l_{1}^{2} l_{2}^{3} m_{2}^{2}+C_{1} C_{C} l_{2}^{3} m_{2}+C_{1} C_{2} m_{1} \\
& +C_{1} C_{2} m_{2}+C_{1} C_{2} m_{C}, \\
p_{3}= & +C_{C} g l_{1}^{3} l_{2}^{2} m_{1} m_{2}+C_{C} g l_{1}^{3} l_{2}^{2} m_{2}^{2}+C_{C} g l_{1}^{2} l_{2}^{3} m_{1} m_{2}+C_{C} g l_{1}^{2} l_{2}^{3} m_{2}^{2} \\
& +C_{2} g l_{1}^{2} m_{1}^{2}+2 C_{2} g l_{1}^{2} m_{1} m_{2}+C_{2} g m_{C} l_{1}^{2} m_{1}+C_{2} g l_{1}^{2} m_{2}^{2}+C_{2} g m_{C} l_{1}^{2} m_{2} \\
& +C_{1} g l_{2}^{2} m_{1} m_{2}+C_{1} g l_{2}^{2} m_{2}^{2}+C_{1} g m_{C} l_{2}^{2} m_{2}+C_{1} C_{2} C_{C}, \\
p_{2}= & g^{2} l_{1}^{2} l_{2}^{2} m_{1}^{2} m_{2}+2 g^{2} l_{1}^{2} l_{2}^{2} m_{1} m_{2}^{2}+m_{C} g^{2} l_{1}^{2} l_{2}^{2} m_{1} m_{2}+g^{2} l_{1}^{2} l_{2}^{2} m_{2}^{3} \\
& +m_{C} g^{2} l_{1}^{2} l_{2}^{2} m_{2}^{2}+C_{2} C_{C} g l_{1}^{2} m_{1}+C_{2} C_{C} g l_{1}^{2} m_{2}+C_{1} C_{C} g l_{2}^{2} m_{2}, \\
p_{1}= & C_{C} g^{2} l_{1}^{2} l_{2}^{2} m_{2}^{2}+C_{C} m_{1} g^{2} l_{1}^{2} l_{2}^{2} m_{2} .
\end{aligned}
$$

Since in typical industrial cranes only the position/speed of the motor (that is, of the cart) is measured, it is convenient to represent (9) as the product of two different transfer functions, namely

$$
F(s)=\frac{X_{P}(s)}{U(s)}=P(s) \frac{1}{s} G(s),
$$

where

$$
P(s):=\frac{V_{C}(s)}{U(s)}=\frac{N_{P}(s)}{D_{P}(s)}
$$

is the transfer function between the force applied to the cart and the velocity of the cart, where

$$
N_{P}(s)=\lambda_{4} s^{4}+\lambda_{3} s^{3}+\lambda_{2} s^{2}+\lambda_{1} s+\lambda_{0},
$$

with

$$
\begin{aligned}
& \lambda_{4}=\frac{l_{1} l_{2} m_{1}}{g^{2}\left(m_{1}+m_{2}\right)}, \\
& \lambda_{3}=\frac{C_{2} l_{1}^{3} m_{1}+C_{1} l_{2}^{3} m_{2}+C_{2} l_{1}^{3} m_{2}}{g^{2} l_{1}^{2} l_{2}^{2} m_{2}\left(m_{1}+m_{2}\right)}, \\
& \lambda_{2}=\frac{g l_{1}^{3} l_{2}^{2} m_{2}^{2}+g m_{1} l_{1}^{3} l_{2}^{2} m_{2}+g l_{1}^{2} l_{2}^{3} m_{2}^{2}+g m_{1} l_{1}^{2} l_{2}^{3} m_{2}+C_{1} C_{2}}{g^{2} l_{1}^{2} l_{2}^{2} m_{2}\left(m_{1}+m_{2}\right)}, \\
& \lambda_{1}=\frac{C_{2} l_{1}^{2} m_{1}+C_{1} l_{2}^{2} m_{2}+C_{2} l_{1}^{2} m_{2}}{g l_{1}^{2} l_{2}^{2} m_{2}\left(m_{1}+m_{2}\right)}, \\
& \lambda_{0}=1,
\end{aligned}
$$


and

$$
D_{P}(s)=D_{F}(s),
$$

and

$$
G(s):=\frac{X_{P}(s)}{X_{C}(s)}=\frac{V_{P}(s)}{V_{C}(s)}=\frac{N_{F}(s)}{D_{P}(s)}
$$

is the transfer function between the position (velocity) of the cart and the position (velocity) of the payload.

\section{Input-Output inversion}

The input-output inversion methodology is described in details in [44]. In brief, the desired output trajectory is defined as the normalized transition polynomial function

$$
\bar{y}(t, \tau)= \begin{cases}0 & t \leq 0 \\ \frac{(2 k+1) !}{k ! \tau^{2 k+1}} \sum_{i=0}^{k} \frac{(-1)^{k-i} t^{2 k-i+1}}{i !(k-i) !(2 k-i+1)} & 0<t<\tau \\ 1 & t \geq \tau .\end{cases}
$$

where $\tau$ is the transition time. The analytical form of the input that results in (14) is then calculated as

$$
W(s ; \tau)=H^{-1}(s) \bar{Y}(s ; \tau),
$$

where $H^{-1}(s)$ is the inverse of the system transfer function and $\bar{Y}(s ; \tau)$ is the Laplace transform of (14).

\section{Control scheme}

A major advantage of the input-output inversion technique is the possibility to decompose the transfer function of the system and to invert the obtained transfer functions separately. This is very useful when the control system allows a feedforward signal to be implemented.

Two control schemes can be used to control the crane, depending on the required task. If a constant velocity is required, the control scheme shown in Figure 2(a) is employed. The cart velocity command signal $\widetilde{v}$ to be applied as input of the closedloop control system can be determined using the input-output inversion technique on the transfer function (13) from the cart velocity to the payload velocity. The cart velocity is measured and the cart velocity is controlled by using a PI controller. The force feedforward signal $\widetilde{u}_{v}$ is then obtained by inverting the transfer function between the force acting on the cart and the velocity of the payload, obtained from (10):

$$
I(s)=\frac{V_{P}(s)}{U(s)}=P(s) G(s) .
$$




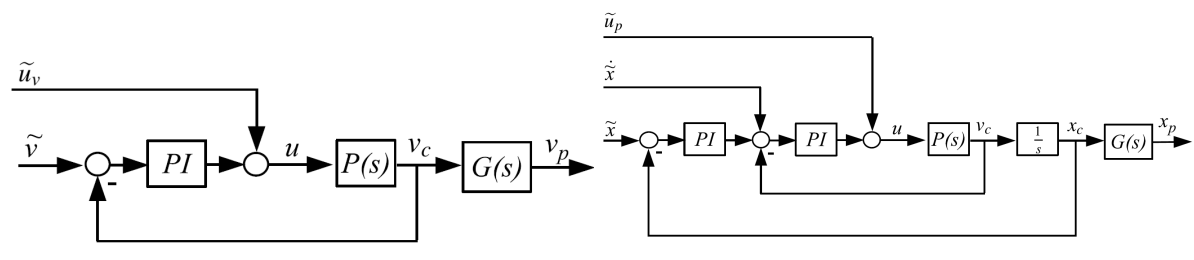

(a)

(b)

Figure 2: (a) Velocity control scheme and (b) position control scheme.

In the case where a given displacement of the payload is desired, the position control scheme in Figure 2(b) is used. A cascade control scheme is then employed with an inner cart velocity control loop and an outer cart position control loop, as customary in industrial motion control applications. The position command signal $\tilde{x}$ is obtained by inverting the transfer function $(13)$ between the cart position and the payload position. Then two different feedforward signals are used in the control system. The cart velocity feedforward signal $\dot{\widetilde{x}}$ is obtained by differentiating the position signal $\widetilde{x}$, while the force feedforward signal $\widetilde{u}_{p}$ is calculated by inverting the transfer function 9 between the force applied to the cart and the position of the payload.

The force feedforward signals $\widetilde{u}_{v}$ and $\widetilde{u}_{p}$, and the velocity feedforward signal $\dot{\tilde{x}}$ increase the tracking performance of the control system. Industrial off-the-shelf drives usually by default allow the implementation of a feedforward signal (at least for the force). Most control techniques for residual oscillation reduction do not provide a force feedforward signal, decreasing the tracking performance, which only relies on the feedback controllers.

\section{Inversion of the Crane Dynamics}

The input-output inversion technique presented in Section 3 is now applied to invert the crane model developed in Section 2 , in order to calculate the command and feedforward signals of the control schemes of Figures 2(a) and Figure 2(b),

\subsection{Cart position (velocity) to payload position (velocity) inversion}

The transfer function (13) describes the relation between the position of the cart and the position of the payload. Its inverse can be expressed as

$$
G^{-1}(s)=\gamma_{2} s^{2}+\gamma_{1} s+\gamma_{0}+\frac{\rho_{1} s+\rho_{0}}{N_{F}(s)},
$$

where $\gamma_{2}, \gamma_{1}, \gamma_{0}, \rho_{1}, \rho_{0}$ are constants that depend on the parameters of the system. Having defined the unitary load trajectory (14), the desired trajectory for a displacement of $q$ can be obtained as $\bar{x}_{p}(t ; \tau)=q \bar{y}(t ; \tau)$. Remembering that (13) is also the transfer function between the cart velocity and the load velocity, the same 
procedure can be applied to determine the cart velocity by defining the desired load speed as $\bar{v}_{p}(t ; \tau)=q \bar{y}(t ; \tau)$. Using (17), the corresponding trajectory of the cart $\widetilde{x}(t ; \tau)$ such that $\left(\widetilde{x}(t ; \tau), \bar{x}_{p}(t ; \tau)\right)$ is an input-output pair for $(13)$ can be calculated, and the cart trajectory is unique and bounded [44].

Defining the inverse Laplace transform of the zero dynamics of the system as

$$
\eta_{0}(t)=\mathscr{L}^{-1}\left\{\frac{\rho_{1} s+\rho_{0}}{N_{F}(s)}\right\}
$$

from (17) we obtain

$$
\widetilde{x}(t ; \tau)=\gamma_{2} \bar{x}_{p}^{(2)}(t ; \tau)+\gamma_{1} \bar{x}_{p}^{(1)}(t ; \tau)+\gamma_{0} \bar{x}_{p}(t ; \tau)+\int_{0}^{t} \eta_{0}(t-v) \bar{y}(v, t) d v .
$$

The determination of $\widetilde{x}(t ; \tau)$ involves the calculation of both the derivative of the polynomial (14) and the convolution integral. The formers can be obtained by explicitly deriving $[14)$ :

$$
D^{\alpha} \bar{y}(t, \tau)= \begin{cases}0 & t \leq 0 \\ \frac{(2 k+1) !}{k ! \tau^{2 k+1}} \sum_{i=0}^{k} \frac{(-1)^{k-i} \tau^{i}(2 k-i+1)}{i !(k-i) !(2 k-i+1)(2 k-i+1-\alpha) !} \tau^{i} t^{2 k-i+1-\alpha} & 0<t<\tau \\ 1 & t \geq \tau .\end{cases}
$$

with $\alpha \in \mathbb{N}$, while the latter can be computed by solving the integral by part. However, when the zero dynamics of the system exhibits a fast decay (namely much faster than the system poles), it can be neglected, as its contribution is filtered by the slow poles of the system.

The zeros of the crane are given by the zeros of the polynomial $N_{F}(s)$ in $(9)$, namely

$$
\begin{aligned}
& z_{1}=-\frac{g l_{1}^{2}\left(m_{1}+m_{2}\right)}{C_{1}}, \\
& z_{2}=-\frac{g l_{2}^{2} m_{2}}{C_{2}} .
\end{aligned}
$$

From (21) it can be seen that the smaller the values of the pivot friction coefficients $C_{1}, C_{2}$, the higher the frequencies at which the zeros occur. From a physical point of view, this means that if the load frictions $C_{1}, C_{2}$ are small, the zero dynamics can be neglected with respect to the dynamics of the system, which implies that the convolution integral $\int_{0}^{t} \eta_{0}(t-v) \bar{y}(v, t) d v$ is also negligible. In other words, the so-called post-action (or post-actuation) [46] can be avoided when controlling systems like overhead cranes, which are characterized by very small friction coefficients $C_{1}, C_{2}$.

This simplification is a major advantage from the control point of view. The negligibility of the convolution integral in (19) implies that the inversion of the system dynamics only involves the derivatives of the desired output. Being the output defined as (14), its derivatives are zero for $t \geq \tau$. This means that the corresponding input is constant for $t \geq \tau$, which implies that the control signal does not change 
after the time $\tau$ has elapsed (see Sections 6 and 9 ).

Thus, if the frictions coefficients $C_{1}, C_{2}$ are small enough, for the input-output inversion purpose only, the transfer function (13) can be approximated as

$$
G(s) \approx \frac{K}{D_{G}(s)},
$$

where $K=g^{2} l_{1}^{2} l_{2}^{2} m_{2}\left(m_{1}+m_{2}\right)$. The inversion of the simplified transfer function (22) gives the simplified polynomial trajectory of the cart

$$
\widetilde{x}(t ; \tau)=\frac{1}{K}\left(\lambda_{4} \bar{x}_{p}^{(4)}(t ; \tau)+\lambda_{3} \bar{x}_{p}^{(3)}(t ; \tau)+\lambda_{2} \bar{x}_{p}^{(2)}(t ; \tau)+\lambda_{1} \bar{x}_{p}^{(1)}(t ; \tau)+\lambda_{0} \bar{x}_{p}(t ; \tau)\right) .
$$

The previous procedure can be applied straightforwardly also to determine the cartto-load speed inverting signal in the case of velocity control, as (13), and consequently (22), describe both the relation between the positions of cart and payload and the relation between the velocities of cart and payload. To this end, it is sufficient to substitute $\bar{x}_{p}(\cdot)$ with the desired speed $\bar{v}_{p}(\cdot)$ (defined as a transition polynomial) and $\widetilde{x}(\cdot)$ with the cart inverting speed $\widetilde{v}(\cdot)$.

Finally, the order $k$ of the transition polynomial $\bar{x}_{p}(t ; \tau)$ can be selected as $k=3$ while, in the case of velocity control, the order of the transition polynomial $\bar{v}_{p}(t ; \tau)$ can be selected as $k=2$, so that the inverting cart reference position signal $\widetilde{x}(t ; \tau)$ is of order $C^{(1)}$, while the inverting set-point reference $\widetilde{v}(t ; \tau)$ is of order $C^{(0)}$ (continuous).

\subsection{Cart force to payload position (velocity) inversion}

As mentioned before, even if industrial motion controllers have position/velocity feedback control loops, typically a feedforward torque signal can be given to the motor in order to improve the tracking performance. In the case of input-output inversion, a feedforward force signal can be calculated by inverting the transfer function between the force applied to the cart and the desired output trajectory (or velocity profile) of the payload. Obviously, the obtained force signal has to be converted into a torque signal by taking into account the transmission ratio of the particular hardware.

Transfer functions (9) and (13) share the same zeros, so that the simplification introduced by neglecting the post-action caused by the convolution integral, which lead to 22], can be used also in this case. For position control, by applying the simplified inversion procedure to 9 we obtain

$$
\begin{aligned}
\widetilde{u}_{p}(t ; \tau)= & K\left(p_{6} \bar{x}_{p}^{(6)}(t ; \tau)+p_{5} \bar{x}_{p}^{(5)}(t ; \tau)+p_{4} \bar{x}_{p}^{(4)}(t ; \tau) p_{3} \bar{x}_{p}^{(3)}(t ; \tau)\right. \\
& \left.+p_{2} \bar{x}_{p}^{(2)}(t ; \tau)+p_{1} \bar{x}_{p}^{(1)}(t ; \tau)\right) .
\end{aligned}
$$

In the case of velocity control, the transfer function to be inverted is the one between the force applied to the cart and the velocity of the payload (16). The 
feedforward input force signal can be obtained by applying the simplified inversion procedure to 16 , obtaining

$$
\begin{aligned}
\widetilde{u}_{v}(t ; \tau)= & K\left(p_{6} \bar{x}_{p}^{(5)}(t ; \tau)+p_{5} \bar{x}_{p}^{(4)}(t ; \tau)+p_{4} \bar{x}_{p}^{(3)}(t ; \tau) p_{3} \bar{x}_{p}^{(2)}(t ; \tau)\right. \\
& \left.+p_{2} \bar{x}_{p}^{(1)}(t ; \tau)+p_{1} \bar{x}_{p}(t ; \tau)\right) .
\end{aligned}
$$

Remark. Theoretically, the value of the transition time $\tau$ can be selected arbitrarily. In practical cases, however, the actuator limits and the robustness of the system have to be considered in the choice of this value. Nevertheless, there is no lower bound of the transition time imposed by the periods of the oscillatory modes of the system, and this represent a clear advantage with respect to other techniques.

\section{Simulation results}

Simulation results have been obtained using Simscape Multibody [48]. In particular a nonlinear model of a double pendulum crane has been built by considering the nominal values of the parameters listed in Table 1 . The zeros of the system are in $[-588.6,-294.3][\mathrm{Hz}]$ and the poles in

$\left[0,-8.3 \cdot 10^{-5},-0.014 \pm 3.391 i,-0.0029 \pm 1.494 i\right][\mathrm{Hz}]$. It can be seen that the zeros are strongly shifted to the left of the complex plane compared to the poles, justifying the assumption of negligible zero dynamics.

As a first example we consider the velocity control with final velocity $q=5[\mathrm{~m} / \mathrm{s}]$ and transition time $\tau=4.21[s]$.

This value of the transition time has been selected in order to perform a fair comparison with the input shaping methodology as it will be shown hereafter. The application of the input-output inversion methodology yields the command signal $\widetilde{v}(23)$ and the feedforward signal $\widetilde{u}_{v}(25)$ shown in Figure 3, where the desired output (i.e., the transition polynomial (14)) is also shown, along with the system response of the nonlinear model. A comparison with the ZV input shaping technique [27] has been performed by considering the same task. In this case, the reference signal has been selected as a ramp with a transient time equal to $1[s]$. The use of a shaper introduces a delay in the reference signal equal to a multiple of the sum of the semi-periods of the system. In particular, the delay introduced by the $\mathrm{ZV}$ shaper is equal to $0.5\left(T_{1}+T_{2}\right)$, where $T_{1}$ and $T_{2}$ are the periods of the first and second oscillatory modes of the system.

For the considered crane, it can be determined that $T_{1}=4.54[\mathrm{~s}]$ and $T_{2}=1.88[\mathrm{~s}]$, so that the total transient time is equal to $\tau=4.21[\mathrm{~s}]$, which is the same transient time used for the input-output inversion. Results obtained with the input shaping are shown in Figure 4

As a second example we consider a position control task with $q=10[\mathrm{~m}]$ and $\tau=4.21[s]$ The signals $\widetilde{x}, \dot{\widetilde{x}}$ e $\widetilde{u}_{p}$ obtained with the inversion based methodology are shown in Figure 5 together with the obtained results. The comparison with ZV input shaping technique (where the same reasoning related to the transition time made for the velocity case applies) can be evaluated in Figure 6. 

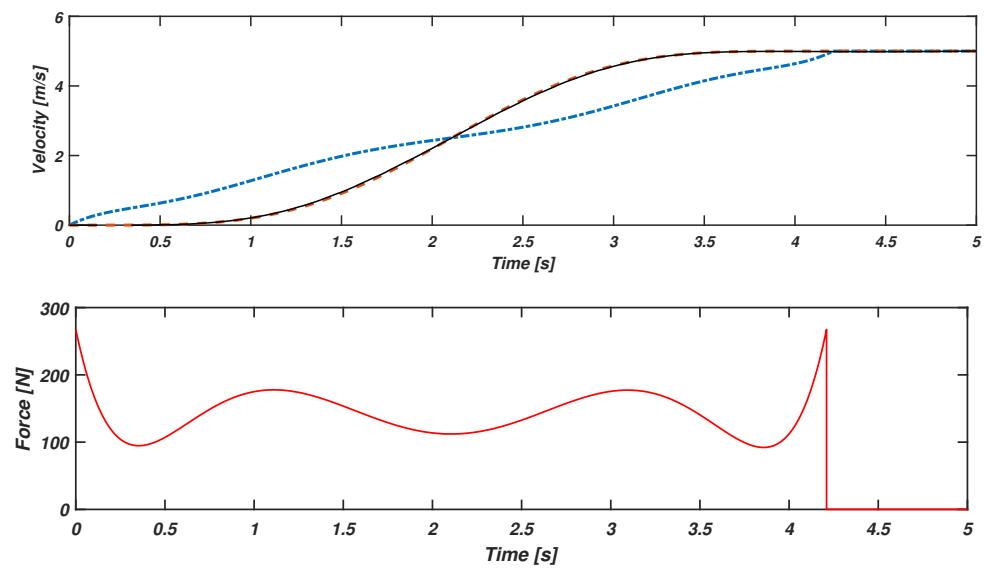

Figure 3: Velocity command signal $\widetilde{v}$ (blue dash-dot line), desired payload trajectory (solid black) and actual payload trajectory obtained with the nonlinear model (orange dashed line) (top). Force feedforward signal $\widetilde{u}_{v}$ for velocity control obtained with input-output inversion of the model (bottom).

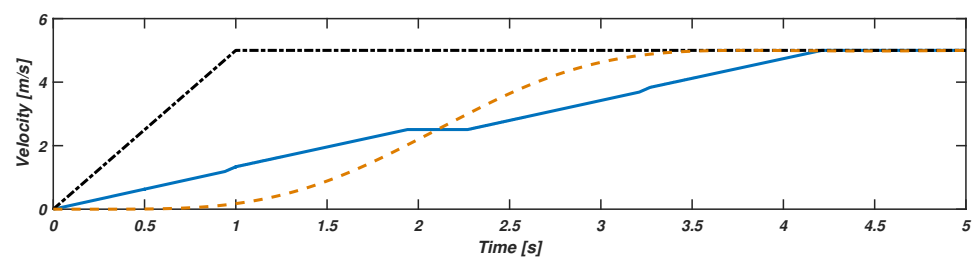

Figure 4: Velocity ramp trajectory reference of the cart (black dash-dot line), shaped cart velocity signal (blue solid line) using the $Z V$ shaper, and actual payload trajectory (dashed orange line). 
Table 1: Parameters of the model used in simulations.

\begin{tabular}{|l|l|l|l|}
\hline \multicolumn{4}{|c|}{ Model data } \\
\hline$m_{C}$ & $100[\mathrm{~kg}]$ & $C_{C}$ & $0.1\left[\frac{\mathrm{Ns}}{\mathrm{m}}\right]$ \\
$m_{1}$ & $10[\mathrm{~kg}]$ & $C_{1}$ & $3\left[\frac{\mathrm{Nms}}{\mathrm{rad}}\right]$ \\
$m_{2}$ & $10[\mathrm{~kg}]$ & $C_{2}$ & $3\left[\frac{\mathrm{Nms}}{\mathrm{rad}}\right]$ \\
$l_{1}$ & $3[\mathrm{~m}]$ & $l_{2}$ & $3[\mathrm{~m}]$ \\
\hline
\end{tabular}
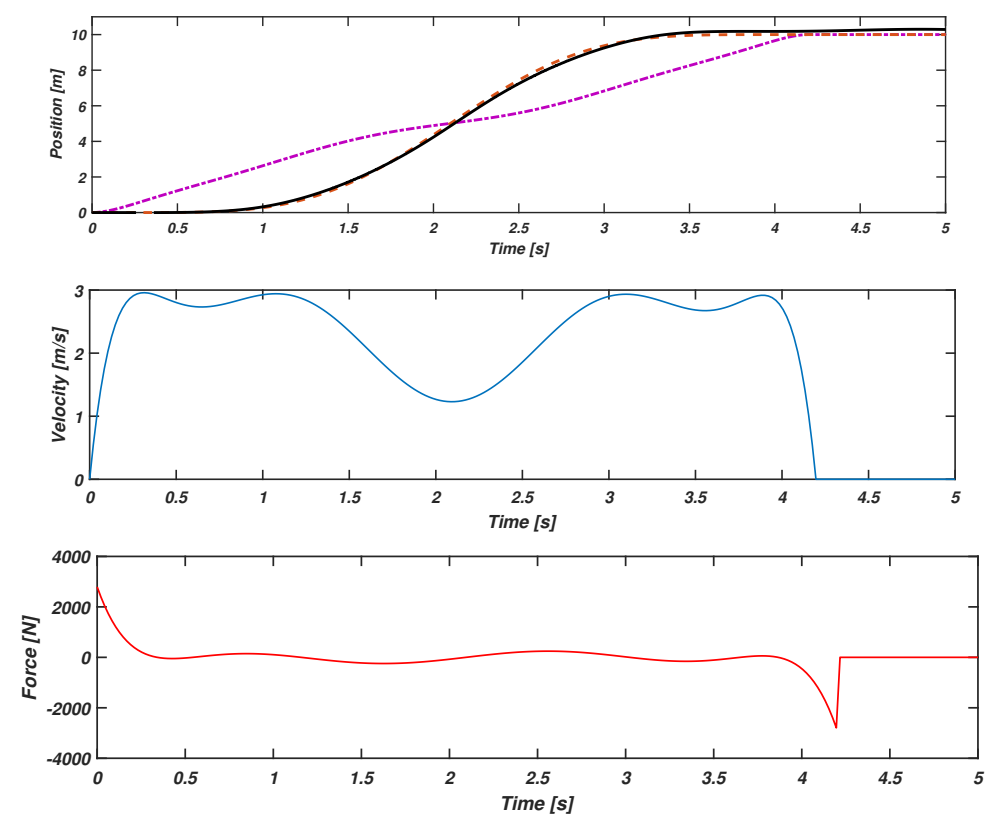

Figure 5: Position commend signal $\widetilde{x}$ (purple dash-dot line), desired payload trajectory (black solid line) and actual payload trajectory (dashed orange line) (top). Cart velocity feedforward signal $\dot{\widetilde{x}}$ (center). Force feedforward signal $\widetilde{u}_{p}$ for position control obtained with input-output inversion of the model (bottom).

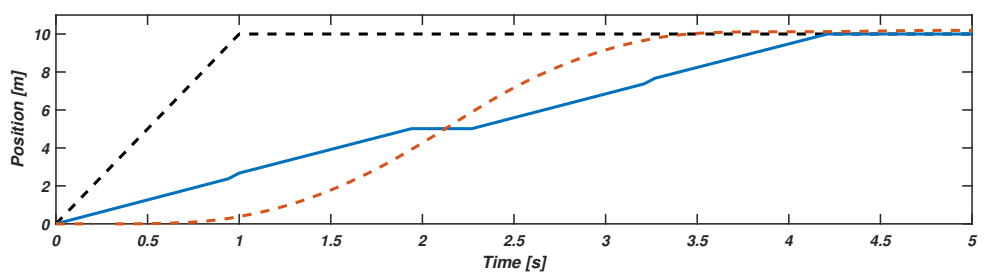

Figure 6: Position trajectory reference of the cart (black dashed line) and shaped cart position signal (blue solid line), using the ZV shaper, and resulting payload trajectory (dashed orange line). 


\section{Robustness}

Robustness is a key issue in open-loop control techniques. In particular, it is interesting to study the robustness of open-loop techniques with respect to structured uncertainties, that is to errors in the model parameters. In the case of gantry cranes, the parameters that are more likely to vary are the length of the hoisting cable $l_{1}$ and the mass of the payload $m_{2}$. In this section the robustness of input-output inversion and input shaping techniques applied to the double pendulum crane model of Section 6 is investigated by means of systematic simulations.

Remark. The measure of robustness adopted in this paper is the sensitivity curves surface, which correlates the maximum residual oscillation of the payload with the parameters uncertainty. The chosen metric is a de facto standard for the evaluation of open-loop techniques [19:23, 25,35, 38], and it is also exploited for the definition of extra-insensitive input shapers [25]. The reason of the success of the maximum residual oscillation for the evaluation of the robustness lies in its simplicity and at-a-glance understandability, which renders the adopted metric very suitable for non highly-specialized engineers and industrial practitioners. This is in line with our objective of devising a industrially feasible solution, easily implementable by using off-the-shelf automation components.

Different input shapers have been applied, namely those listed in Table 2. where, for each case, the associated delays and the resulting total transient times are also shown.

For a fair comparison between input-output inversion and input shaping tech-

Table 2: Delays introduced by the input shaping techniques. $T$ is the sum of the periods of the system to be controlled.

\begin{tabular}{|l|l|l|}
\hline IS technique & Delay & Total transient time $[s]$ \\
\hline ZV & $0.5 T$ & 4.21 \\
ZVD & $T$ & 7.42 \\
EI & $T$ & 7.42 \\
ZVDD & $1.5 T$ & 10.63 \\
Two-hump EI & $1.5 T$ & 10.63 \\
\hline
\end{tabular}

niques, the transient time $\tau$ of the reference polynomial (23) has been set, in each case, equal to the total transient time of input shaping techniques.

The results obtained simulating the response of the system varying $l_{1}$ and $m_{2}$ are plotted in Figure7 The robustness is measured as the maximum value of the residual oscillation, that is the maximum distance of the payload from the equilibrium position after the transient time. First of all, in Figure 7(a) and 7(b) it can be seen that the performance of the input-output inversion technique is comparable with the performance of $\mathrm{ZV}$ input shaping techniques. More robust techniques, such as ZVD, ZVDD, EI IS and Two-hump EI IS have an increased robustness when 


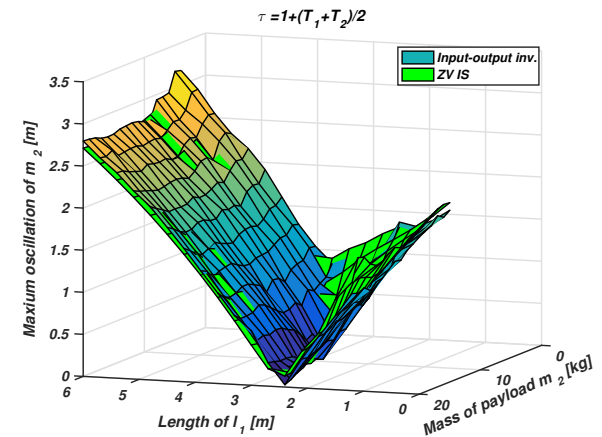

(a) Position control, total time of $1+\frac{T_{1}+T_{2}}{2}$

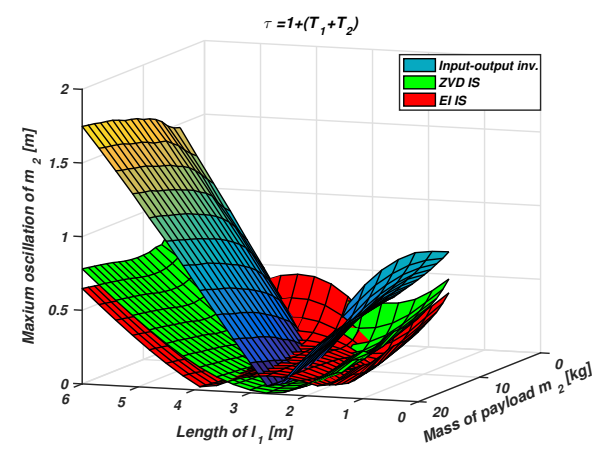

(c) Position control, total time of $1+T_{1}+T_{2}$

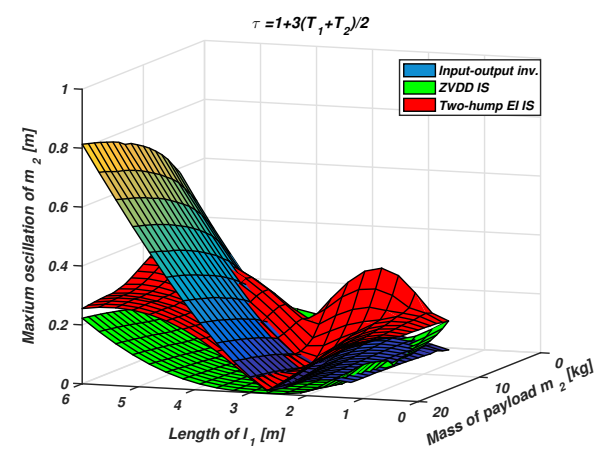

(e) Position control, total time of $1+\frac{3}{2}\left(T_{1}+\right.$ (f) Velocity control, total time of $1+\frac{3}{2}\left(T_{1}+T_{2}\right)$ $\left.T_{2}\right)$

Figure 7: Comparison of the robustness to parameter errors of input shaping and input-output inversion technique for both position (left) and velocity (right) control.

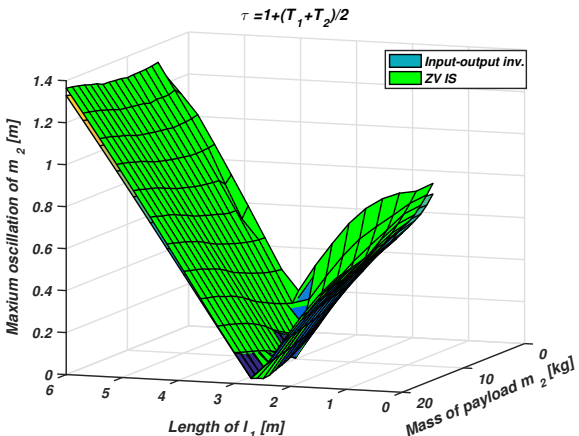

(b) Velocity control, total time of $1+\frac{T_{1}+T_{2}}{2}$

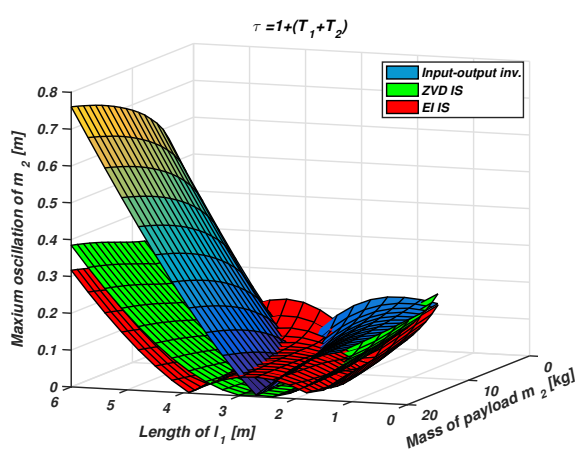

(d) Velocity control, total time of $1+T_{1}+T_{2}$

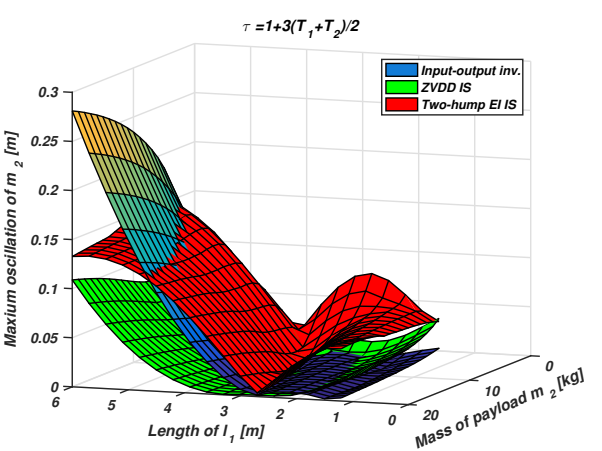

\author{
(1)
}


compared with the input-output inversion technique with the same transient time $\tau$. From the results in Figure 7(c), 7(d), 7(e), 7(f), it can be seen that, even if input shaping techniques are more robust, the input-output inversion methods present a similar behaviour in the region of the correct parameters, i.e., the advantages of input shaping over the input-output inversion can only be appreciated when the modeling errors are extremely high. Further, the input-output inversion performance in terms of robustness is comparable to the one of the two-hump input shaping technique.

These observations are valid for both position and velocity control, as input shaping and input-output inversion have the same response in terms of robustness.

Due to the strong dependence of the frequencies of the system on the length of the cable, open-loop techniques for the control of overhead cranes are generally robust with respect to changes in the mass of the payload, while they are more sensitive to changes in the length of the cable. This dependence is well represented by the V-shape of the graphs in Figure 7. In any case it also appears that the robustness of the input-output inversion technique increases when the transient time increases, which makes the role of this design parameter clear.

\section{Experimental setup}

In order to test the performance and the applicability of the proposed technique, a $3 \times 3[\mathrm{~m}]$ laboratory overhead crane has been used. For the purpose of this paper, the bearing structure can be considered rigid. Two different payloads have been used: a cylindrical concrete block with non-negligible moment of inertia, and two iron discs, linked by means of a cable, that simulates the presence of a hook and a concentrated payload. The laboratory overhead crane used can be seen in Figure 8 with the distributed payload configuration and with the hook and concentrated payload configuration.

For the cylindrical distributed payload like the one used for this setup, an equivalent two-mass pendulum model can be obtained by solving (1), resulting in

$$
\left\{\begin{array}{l}
m_{1}=\frac{1}{4} M \\
m_{2}=\frac{3}{4} M \\
l_{2}=\frac{7}{12} L
\end{array}\right.
$$

where $M$ and $L$ are the mass and the length of the cylindrical payload. The equivalent values of both double and simple pendulum that fully describe the dynamics of the real system are listed in Table 3 . These values have been directly measured (masses and lengths) or experimentally obtained. The corresponding zeros and poles, expressed in $\left[s^{-1}\right]$ are

$$
\begin{aligned}
& Z_{1}=[-1206,-60.8], P_{1}=[0,-1.923,-0.556 \pm 9.840 i,-0.1711 \pm 2.656 i], \\
& Z_{2}=[-529.3,-39.18], P_{2}=[0,-1.957,-0.266 \pm 5.728 i,-0.157 \pm 2.622 i] .
\end{aligned}
$$



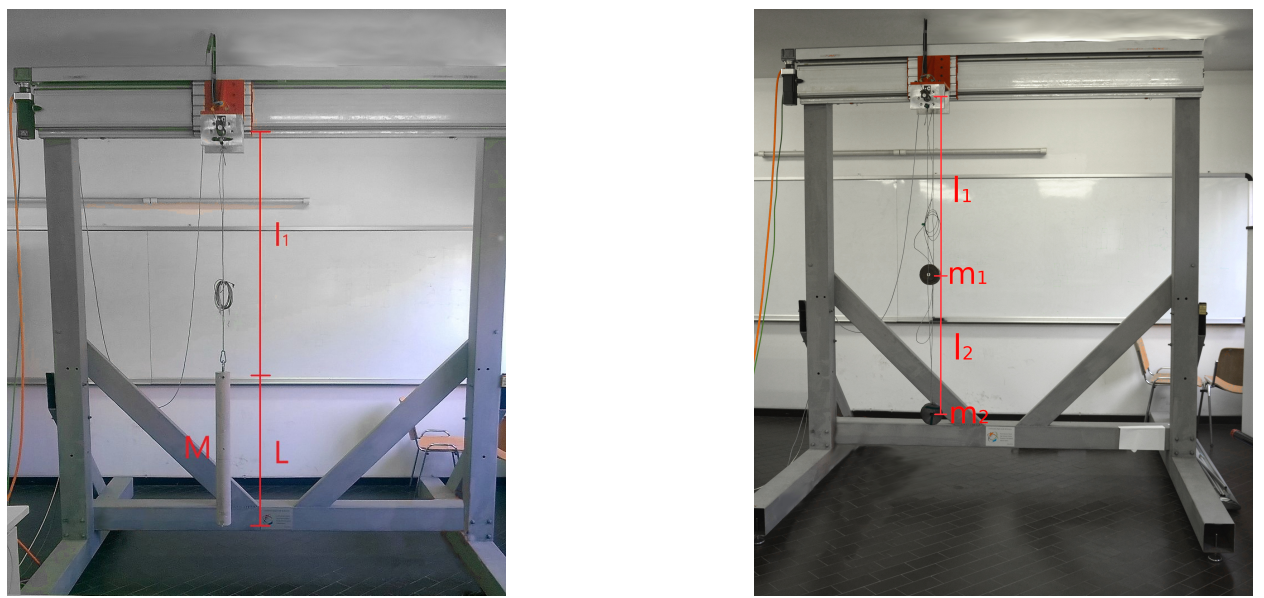

Figure 8: The 2D laboratory overhead crane used for the experimental evaluation of the method proposed. Distributed payload (left) and hook and concentrated payload (right) cofigurations.

Table 3: Parameters of the crane model with distributed payload and hook plus concentrated payload.

\begin{tabular}{|l|c|c|}
\hline & Distributed payload & Hook plus payload \\
\hline$m_{C}$ & $38.0[\mathrm{~kg}]$ & $38.0[\mathrm{~kg}]$ \\
$m_{1}$ & $2.45[\mathrm{~kg}]$ & $4.57[\mathrm{~kg}]$ \\
$m_{2}$ & $7.35[\mathrm{~kg}]$ & $3.78[\mathrm{~kg}]$ \\
$l_{1}$ & $1.12[\mathrm{~m}]$ & $1.03[\mathrm{~m}]$ \\
$l_{2}$ & $0.581[\mathrm{~m}]$ & $0.86[\mathrm{~m}]$ \\
$M$ & $9.80[\mathrm{~kg}]$ & $8.35[\mathrm{~kg}]$ \\
$L$ & $1.556[\mathrm{~m}]$ & $1.419[\mathrm{~m}]$ \\
$C_{C}$ & $85[\mathrm{Ns} / \mathrm{m}]$ & $85[\mathrm{Ns} / \mathrm{m}]$ \\
$C_{1}$ & $0.1[\mathrm{Nms} / \mathrm{rad}]$ & $0.15[\mathrm{Nms} / \mathrm{rad}]$ \\
$C_{2}$ & $0.4[\mathrm{Nms} / \mathrm{rad}]$ & $0.7[\mathrm{Nms} / \mathrm{rad}]$ \\
\hline
\end{tabular}




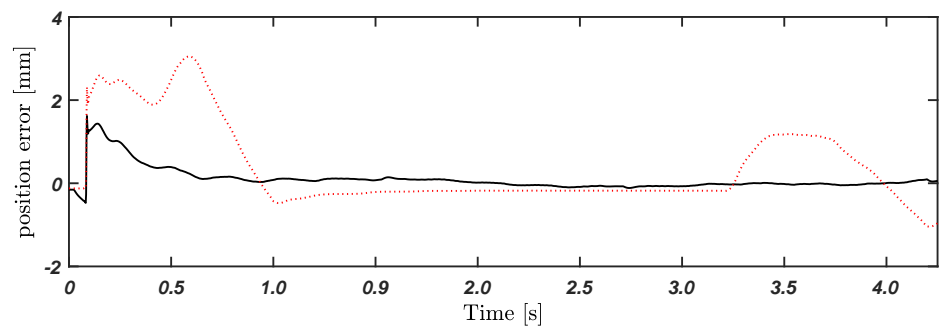

Figure 9: Cart position tracking error during a displacement of $1.2[\mathrm{~m}]$ using inputoutput inversion technique, with (solid line) and without (dotted line) the torque feedforward.

It can be seen that the zeros of the system exhibits a faster dynamics with respect to the poles, justifying the assumption of negligible post action.

The control system has been implemented by means of a standard industrial PLC. In particular, the position and velocity control systems in Figure 2(a) and Figure 2(b), respectively, are embedded into the motor drive; the PI controllers have been tuned by a trial-and-error procedure. The use of a torque feedforward and the slow dynamics of the crane justify a non-optimal tuning of the PI controllers, provided that the reference trajectory is followed with a satisfactory tracking performance (note that this is almost always the case in industry). Indeed, using a torque feedforward, the PI controllers only compensate for the possible mismatch between the model and the real crane, and, at least in the nominal case, they could be ignored. In any case, even without using the torque feedforward, the obtained tracking error is negligible, as shown in Figure 9, as evidence of the good tuning of the controllers. If then we use the torque feedforward, the dynamic tracking error becomes virtually zero, and the only source of (negligible) tracking error remains the unmodeled passage from static to kinetic friction (clearly visible at the beginning of the motion task in Figure 9p. Moreover, another torque feedforward signal compensates the nonlinearity introduced by the Coulomb friction that affects the sliding of the cart in our setup. The parametric command signals described by (23), ( $\widetilde{v}$ and $\widetilde{x}$ in Figure 2(a) and Figure 2(b) respectively), and (24) and 25), ( $\widetilde{u}_{v}$ and $\widetilde{u}_{p}$ in the same figures) are generated by means of function blocks written in IEC 61131-3 structured text. The feedforward velocity signal $\dot{\tilde{x}}$ in Figure 2(b) is automatically calculated by the servo drive numerically differentiating the noisefree signal $\widetilde{x}$.

It is worth stressing that the system has been assembled using off-the-shelf industrial components. The presented method results in a computationally light polynomial reference signal, that can be calculated in closed form, and it is thereby implementable in virtually every commercially available servo drive and PLC. Moreover, the obtained reference signal (13) is parametrized in $\tau$, that is, the total transition time, and in the parameters of the model in Figure 1, which are easy to measure. 
The angles during the tests have been measured using a narrow-band camera that acquires markers positioned on the payload and reconstructing frame by frame the angles $\theta_{1}$ and $\theta_{2}$ of the model in Figure [1 [35]. Note that this measurement has never been employed in the control algorithm, but its only purpose is to evaluate the achieved performance.

\section{Experimental results}

Experimental results obtained with the setup described in Section 8 are presented and discussed in this section. In particular, the effectiveness of the simplified inputoutput inversion technique for double pendulum cranes, is experimentally demonstrated.

\subsection{Velocity control}

For the velocity control task, the payload is required to move from zero velocity to a velocity of $q=500[\mathrm{~mm} / \mathrm{s}]$ with different transient times. For the distributed payload, which have periods $T_{1}=2.36[s]$ and $T_{2}=0.64[s]$, a ramp reference signal with an acceleration of $1720\left[\mathrm{~mm} / \mathrm{s}^{2}\right]$, considering the delay introduced by the use of $Z V$ technique in Table 2, gives a total transient time of $\tau=1.79$ [s]. For the hook plus payload setup, the values of the parameters in Table 3 give periods of $T_{1}=2.38[\mathrm{~s}]$ and $T_{2}=1.01[\mathrm{~s}]$. A ramp with an acceleration of $4300\left[\mathrm{~mm} / \mathrm{s}^{2}\right]$ gives a transient time $\tau=1.81[s]$. The tests have been executed using input shaping multimode $Z V$ technique and input-output inversion technique based both on simple and double pendulum models. The results in terms of residual oscillations can be seen in Figure 10. The displacement from the nominal position after the movement when anti-sway techniques are not used reaches $0.06[\mathrm{~m}]$ in the case of distributed payload and $0.10[\mathrm{~m}]$ in the case of hook and point mass payload.

The transient time has then been reduced to $1.2[s]$. This is not feasible with the input shaping, as for both the setups the use of input shaping techniques introduces a delay (listed in Table 2) that is more than $1.2[s]$. The results applying input-output inversion technique based both on the simple and the double pendulum model for a transient time of $\tau=1.2[s]$ are shown in Figure 11. In this case, the displacement from the nominal position after the movement when anti-sway techniques are not used reaches $0.03[\mathrm{~m}]$ in the case of distributed payload and $0.12[\mathrm{~m}]$ in the case of hook and pointmass payload.

\subsection{Position control}

Input-output inversion and input shaping techniques have been applied also to the position control of the system. In particular, the payload has been moved for a total displacement of $1.2[\mathrm{~m}]$ in the transient times $\tau=4$ and $\tau=3[\mathrm{~s}]$. Given a maximum acceleration of $3000\left[\mathrm{~mm} / \mathrm{s}^{2}\right]$ and a trapezoidal law of motion for the 

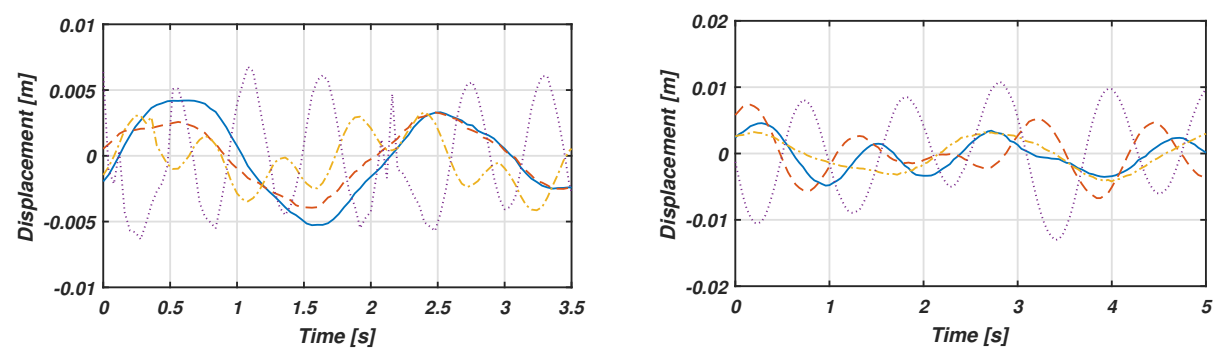

Figure 10: Residual oscillations in the case of velocity control for the distributed payload (left) with $\tau=1.79[s]$ and the hook and pointmass payload (right) with $\tau=1.81[s]$ using input shaping technique based on a simple (dotted violet line) and double (dash-dot yellow line) pendulum model and using input output inversion technique based on a simple (dashed red line) and double (solid blue line) pendulum model.
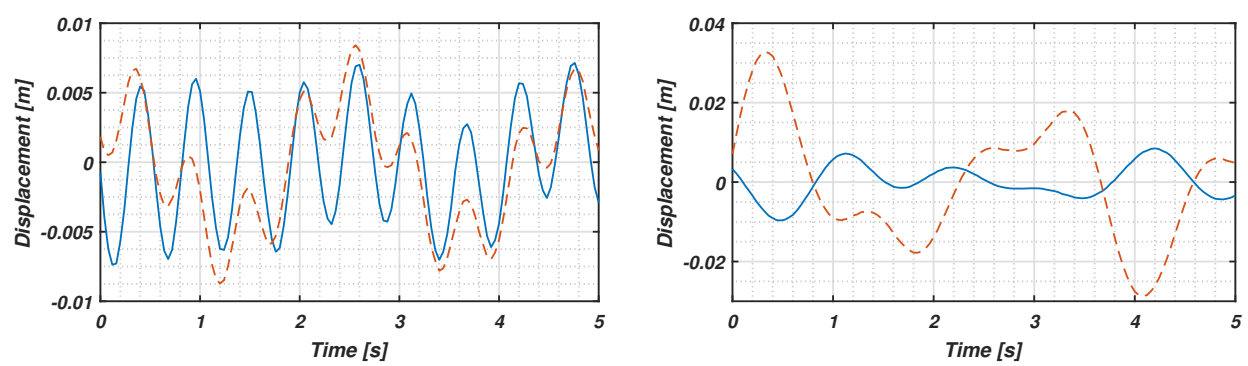

Figure 11: Residual oscillations in the case of velocity control for the distributed payload (left) and the hook and pointmass payload (right) with $\tau=1.20[s]$ using input output inversion technique based on a simple (dashed red line) and double (solid blue line) pendulum model. 

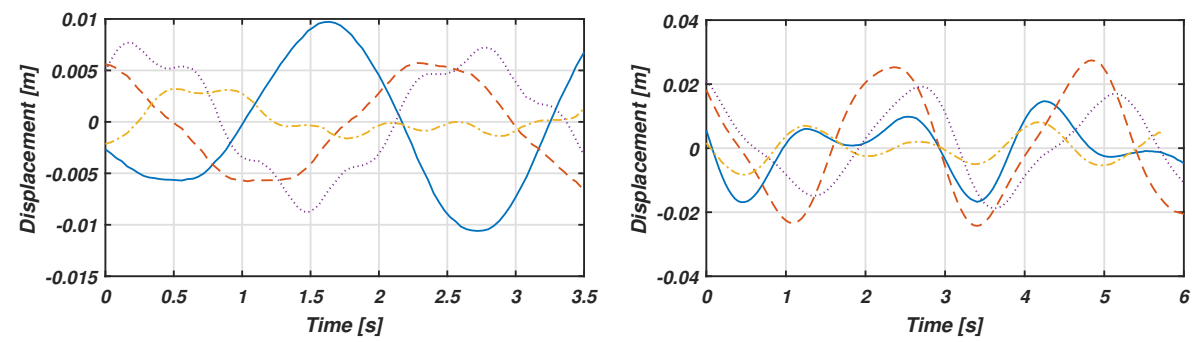

Figure 12: Residual oscillations in the case of position control for the distributed payload (left) and the hook and pointmass payload (right) with $\tau=4[s]$ using input shaping technique based on a simple (dotted violet line) and double (dashdot yellow line) pendulum model and using input output inversion technique based on a simple (dashed red line) and double (solid blue line) pendulum model.
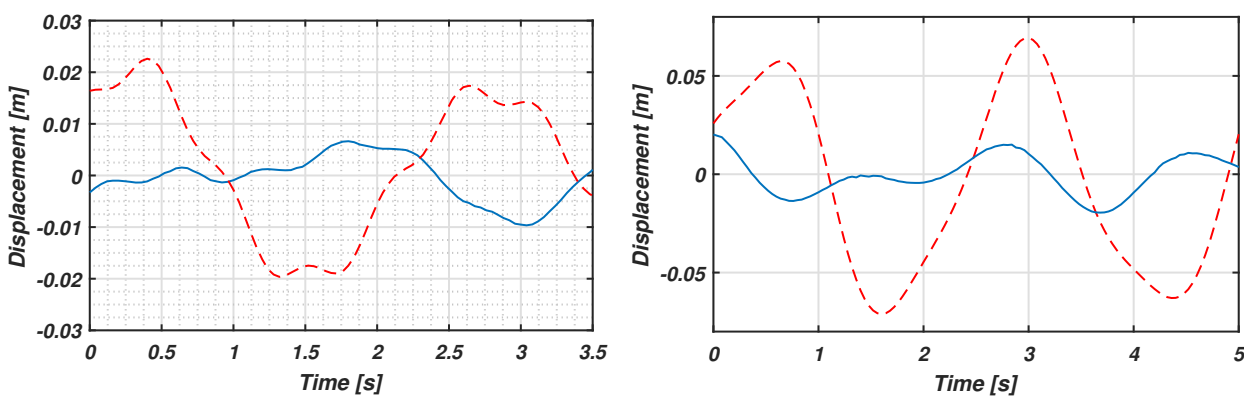

Figure 13: Residual oscillations in the case of position control for the distributed payload (left) and the hook and pointmass payload (right) with $\tau=3[s]$ using input output inversion technique based on a simple (dashed red line) and double (solid blue line) pendulum model.

input shaping control, only the transient time of $4[s]$ is feasible for the input shaping technique. The results in terms of residual oscillations are in Figure 12. The displacement from the nominal position when anti-sway techniques are not used reaches $0.32[\mathrm{~m}]$ in the case of distributed payload and $0.29[\mathrm{~m}]$ in the case of hook and point mass payload.

The transient time has then been reduced to $3[s]$, which is not feasible with the input shaping. The results obtained with the input-output inversion technique, based both on the simple and the double pendulum model, for a transient time of $\tau=3[s]$ are shown in Figure 13. In this case, the displacement from the nominal position after the movement when anti-sway techniques are not used reaches $0.13[\mathrm{~m}]$ in the case of distributed payload and $0.17[\mathrm{~m}]$ in the case of hook and concentrated payload. 


\subsection{Results analysis}

From the analysis of the experimental results of both velocity and position control, it can be seen that the use of a double pendulum model in an anti sway technique leads to superior performance. The improvement in performance is more significant, for both the distributed payload and the hook and point mass payload, when the transient time $\tau$ decreases. With a decreased transient time, in fact, the high frequency-components of the system are more excited, and they affect more the response of the system. While a simple pendulum model is able to describe only the main frequency of the crane, the double pendulum model also captures the second oscillation mode, higher in frequency, yielding small residual oscillations also in fast movements.

By analyzing the results in Figure 11 and in Figure 13 it appears that the inputoutput inversion guarantees a significant improvement in the performance compared to the one obtained without anti-sway techniques, even when, due to the reduced transient time, input shaping techniques are not implementable.

\section{Conclusions}

In this paper, we propose an input-output inversion technique for the reduction of residual oscillations of overhead cranes modelled as a double pendulum. Both velocity and position control tasks have been considered.

The method can be easily implemented with commercial off-the-shelf industrial components, thanks to the provided analysis that shows that the postaction can be neglected, which allows the analytical determination of the inversion-based command as a simple parametric polynomial. A relevant feature of the technique is that the transient time $\tau$ of the desired output function is a design parameter that can be selected in order to fully exploit the actuator capabilities and to handle the trade-off between performance and robustness.

Simulation and experimental results have shown the effectiveness of the proposed methodology in handling those situations where the mass of the hook is significant with respect to the mass of the payload or, equivalently, when there is a distributed payload. A comparison with the input shaping technique has shown that the two techniques provide a similar robustness. However, the inversion-based method does not constrain the minimum transient time to be a function of the sum of the periods of the system.

\section{Acknowledgements}

This work is partially developed within the European Union H2020 program ECSEL2016-1 under grant agreement n. 737453 (I-MECH). 


\section{References}

[1] E. M. Abdel-Rahman, A. H. Nayfeh, and Z. N. Masoud, "Dynamics and control of cranes: A review," Modal Analysis, vol. 9, no. 7, pp. 863-908, 2003.

[2] X. Zhang, Y. Fang, and N. Sun, "Minimum-time trajectory planning for underactuated overhead crane systems with state and control constraints," IEEE Transactions on Industrial Electronics, vol. 61, no. 12, pp. 6915-6925, 2014.

[3] B. Kolar, H. Rams, and K. Schlacher, "Time-optimal flatness based control of a gantry crane," Control Engineering Practice, vol. 60, pp. 18-27, 2017.

[4] J. W. Auernig and H. Troger, "Time optimal control of overhead cranes with hoisting of the load," Automatica, vol. 23, pp. 437-447, 1987.

[5] N. Sun and Y. Fang, "New energy analytical results for the regulation of underactuated overhead cranes: an end-effector motion-based approach," IEEE Transactions on Industrial Electronics, vol. 59, pp. 4723-4734, 2012.

[6] X. Wu and X. He, "Enhanced damping-based anti-swing control method for underactuated overhead cranes," IET Control Theory and Applications, vol. 9, pp. 1893-1900, 2015.

[7] A. Benhidjeb and G. L. Gissinger, "Fuzzy control of an overhead crane performance comparison with classic control," Control Engineering Practice, vol. 3, pp. 1687-1696, 1995.

[8] M.-S. Park, D. Chwa, and S.-K. Hong, "Antisway tracking control of overhead cranes with system uncertainty and actuator nonlinearity using an adaptive fuzzy sliding-mode control," IEEE Transactions on Industrial Electronics, vol. 55, pp. 3972-3984, 2008.

[9] Y. Zhao and H. Gao, "Fuzzy-model-based control of an overhead crane with input delay and actuator saturation," IEEE Transactions on Fuzzy Systems, vol. 9, pp. 1893-1900, 2012.

[10] L. V. den Broeck, M. Diehl, and J. Swevers, "A model predictive control approach for time optimal point-to-point motion control," Mechatronics, vol. 21, pp. 1203-1212, 2011.

[11] B. Käpernick and K. Graichen, "Model predictive control of an overhead crane using constraint substitution," in Proceedings American Control Conference, 2013, pp. 3973-3978.

[12] J. Smoczek, "Experimental verification of a GPC-LPV method with RLS and P1-TS fuzzy-based estimation for limiting the transient and residual vibration of a crane system," Mechanical Systems and Signal Processing, vol. 62-63, pp. 324-340, 2015. 
[13] M. Ermidoroa, A. Cologni, S. Formentin, and F. Previdi, "Fixed-order gain-scheduling anti-sway control of overhead bridge cranes," Mechatronics, vol. 39, pp. 237-247, 2016.

[14] K. Zavari, G. Pipeleers, and J. Swevers, "Gain-scheduled controller design: illustration on an overhead crane," IEEE Transactions on Industrial Electronics, vol. 61, pp. 3713-3718, 2014.

[15] Q. H. Ngo and K.-S. Hong, "Sliding-mode antisway control of an offshore container crane," IEEE/ASME Transactions on Mechatronics, vol. 17, pp. 201-209, 2012.

[16] L. A. Tuan, S.-G. Lee, D. H. Ko, and L. C. Nho, "Combined control with sliding mode and partial feedback linearization for $3 \mathrm{~d}$ overhead cranes," International Journal of Robust and Nonlinear Control, vol. 24, pp. 3372-3386, 2014.

[17] N. Sun, Y. Fang, H. Chen, B. Lu, and Y. Fu, "Slew/translation positioning and swing suppression for 4-dof tower cranes with parametric uncertainties: Design and hardware experimentation," IEEE Transactions on Industrial Electronics, vol. 63, no. 10, pp. 6407-6418, 2016.

[18] J. Huang, X. Xie, and Z. Liang, "Control of bridge cranes with distributedmass payload dynamics," IEEE/ASME Transactions on Mechatronics, vol. 20, pp. 481-486, 2015.

[19] N. C. Singer and W. P. Seering, "Preshaping command inputs to reduce system vibration," Journal of Dynamic Systems, Measurement, and Control, vol. 112, no. 1, pp. 76-82, 1990.

[20] J. J. Potter and W. Singhose, "Improving manual tracking of systems with oscillatory dynamics," IEEE Transactions on Human-Machine Systems, vol. 43, pp. 46-52, 2013.

[21] T. Singh and G. R. Heppler, "Shaped input control of a system with multiple modes," ASME Journal of Dynamic Systems, Measurement, and Control, vol. $115,1993$.

[22] W. Singhose, E. Crain, and W. Seering, "Convolved and simultaneous twomode input shapers," IEE Proceedings - Control Theory and Applications, vol. 144 , no. 6 , pp. 515-520, 1997.

[23] W. Singhose, W. Seering, and N. Singer, "Residual vibration reduction using vectors diagrams to generate shaped inputs," Journal of Mechanical Design, vol. 116, no. 2, pp. 654-659, 1994.

[24] W. E. Singhose, L. J. Porter, T. D. Tuttle, and N. C. Singer, "Vibration reduction using multi-hump input shapers," ASME Journal of Dynamic Systems, Measurement, and Control, vol. 119, pp. 320-326, 1997. 
[25] J. Vaughan, A. Yano, and W. Singhose, "Comparison of robust input shapers," Journal of Sound and Vibration, vol. 315, pp. 797-815, 2008.

[26] S. Garrido, M. Abderrahim, A. Gimenez, R. Diez, and C. Balaguer, "Antiswinging input shaping control of an automatic construction crane," IEEE Transactions on Automation Science and Engineering, vol. 5, pp. 549-557, 2008.

[27] W. Singhose, "Command shaping for flexible systems: A review of the first 50 years," International Journal of Precision Engineering and Manufacturing, vol. 10, no. 4, pp. 153-168, 2009.

[28] W. Singhose and J. Vaughan, "Reducing vibration by digital filtering and input shaping," IEEE Transactions on Control Systems Technology, vol. 19, pp. 1410-1420, 2011.

[29] Z. Wu and X. Xia, "Optimal motion planning for overhead cranes," IET Control Theory and Applications, vol. 8, pp. 1833-1842, 2014.

[30] D. Liu, W. Guo, and J. Yi, "Dynamics and GA-based stable control for a class of underactuated mechanical systems," International Journal of Control, Automation, and Systems, vol. 6, no. 1, pp. 35-43, 2008.

[31] N. Sun, Y. Wu, Y. Fang, and H. Chen, "Nonlinear antiswing control for crane systems with double-pendulum swing effects and uncertain parameters: design and experiments," IEEE Transactions on Automation Science and Engineering, 2017.

[32] D. Qian, S. Tong, and S. Lee, "Fuzzy-logic-based control of payloads subjected to double-pendulum motion in overhead cranes," Automation in Construction, vol. 65, pp. 133-143, 2016.

[33] L. A. Tuan and S.-G. Lee, "Sliding mode controls of double-pendulum crane systems," Journal of Mechanical Science and Technology, vol. 27, no. 6, pp. 1863-1873, 2013.

[34] N. Sun, Y. Fang, H. Chen, and Y. Fu, "Super-twisting-based antiswing control for underactuated double pendulum cranes," in Proceedings IEEE International Conference on Advanced Intelligent Mechatronics, 2015, pp. 749-754.

[35] J. Vaughan, D. Kim, and W. Singhose, "Control of tower cranes with doublependulum payload dynamics," IEEE Transactions on Control Systems Technology, vol. 18, no. 6, pp. 1345-1358, 2010.

[36] Z. Masoud, K. Alhazza, E. Abu-Nada, and M. Majeed, "A hybrid commandshaper for double-pendulum overhead cranes," Journal of Vibration and Control, vol. 20, no. 1, pp. 24-37, 2014. 
[37] K.-T. Hong, C.-D. Huh, and K.-S. Hong, "Command shaping control for limiting the transient sway angle of crane systems," International Journal of Control, Automation, and Systems, vol. 1, no. 1, pp. 43-53, 2003.

[38] W. E. Singhose, D. Kim, and M. Kenison, "Input shaping control of doublependulum bridge crane oscillations," Journal of Dynamic Systems, Measurement, and Control, vol. 130, no. 3, p. 034504, 2008.

[39] N. Sun, Y. Wu, H. Chen, and Y. Fang, "An energy-optimal solution for transportation control of cranes with double pendulum dynamics: Design and experiments," Mechanical Systems and Signal Processing, vol. 102, pp. 87-101, 2018.

[40] H. Chen, Y. Fang, and N. Sun, "A time-optimal trajectory planning strategy for double pendulum cranes with swing suppression," in Proceedings 35th Chinese Control Conference. IEEE, 2016, pp. 4599-4604.

[41] _ _ "A swing constrained time-optimal trajectory planning strategy for double pendulum crane systems," Nonlinear Dynamics, vol. 89, no. 2, pp. 1513$1524,2017$.

[42] A. Piazzi and A. Visioli, "Minimum-time system-inversion-based motion planning for residual vibration reduction," IEEE/ASME Transactions on Mechatronics, vol. 5, no. 1, pp. 12-22, 2000.

[43] — - "Optimal dynamic-inversion-based control of an overhead crane," IEE Proceedings - Control Theory and Applications, vol. 149, no. 5, pp. 405-411, 2002.

[44] F. Padula, A. Visioli, D. Facchinetti, and A. Saleri, "A dynamic inversion approach for oscillation-free control of overhead cranes," in Proceedings IEEE International Conference on Emerging Technologies and Factory Automation, 2015.

[45] A. Piazzi and A. Visioli, "Optimal noncausal set-point regulation of scalar systems," Automatica, vol. 37, no. 1, pp. 121 - 127, 2001.

[46] H. Perez and S. Devasia, "Optimal output-transitions for linear systems," $A u$ tomatica, vol. 39, no. 2, pp. 181-192, 2003.

[47] Z. N. Masoud, "Effect of hoisting cable elasticity on anti-sway controllers of quay-side container cranes," Nonlinear Dynamics, vol. 58, no. 1-2, p. 129, 2009.

[48] MathWorks. (2016) Simscape ${ }^{\mathrm{TM}}$ multibody ${ }^{\mathrm{TM}}$ getting started guide. http://cn. mathworks.com/help/pdf_doc/physmod/sm/sm_gs.pdf. 